\title{
Virtual states of light non-Borromean halo nuclei
}

\author{
A. Delfino, ${ }^{1}$ T. Frederico, ${ }^{2}$ M. S. Hussein, ${ }^{3}$ and Lauro Tomio ${ }^{4}$ \\ ${ }^{1}$ Departamento de Física, Universidade Federal Fluminense, 24210-340 Niterói, Rio de Janeiro, Brazil \\ ${ }^{2}$ Departamento de Física, Instituto Tecnológico de Aeronáutica, Centro Técnico Aeroespacial, \\ 12228-900 São José dos Campos, Brazil \\ ${ }^{3}$ Nuclear Theory and Elementary Particle Phenomenology Group, Instituto de Física, Universidade de São Paulo, \\ 05315-970, São Paulo, Brazil \\ ${ }^{4}$ Instituto de Física Teórica, Universidade Estadual Paulista, 01405-900 São Paulo, Brazil
}

(Received 22 December 1999; published 30 March 2000)

\begin{abstract}
It is shown that three-body non-Borromean halo nuclei like ${ }^{12} \mathrm{Be},{ }^{18} \mathrm{C},{ }^{20} \mathrm{C}$, considered as neutron-neutroncore systems, have $p$-wave virtual states with energy of about 1.7 times the corresponding neutron-core binding energy. We use a renormalizable model that guarantees the general validity of our results in the context of short range interactions.
\end{abstract}

PACS number(s): 27.20.+n, 21.10.Dr, 21.45.+v, 24.30.Gd

Halo nuclei offer the opportunity to study few-body aspects of the nuclear interaction with their peculiar three-body phenomena. Recently, attention was drawn to the possibility of existing Efimov states [1] in such systems, because some halo nuclei can be viewed as a three-body system, with two loosely bound neutrons and a core [2-4]. It was suggested [5] that ${ }^{18} \mathrm{C}$ and ${ }^{20} \mathrm{C}$ are promising candidates to have Efimov states. In Ref. [6], by considering the critical conditions to allow the existence of one Efimov state and using the experimental values for the neutron separation energies $\left({ }^{19} \mathrm{C}+n\right.$ and $\left.{ }^{18} \mathrm{C}+2 n\right)$ given in Ref. [7], it was concluded that ${ }^{20} \mathrm{C}$ could have such a state.

The weakly bound Efimov states [1] appear in the zero angular momentum state of a three boson system. The number of states, condensing at zero energy, grows to infinity as the pair interactions are just about to bind two particles in the $s$ wave. Such states are loosely bound and their wave functions extend far beyond those of normal states. If such states exist in nature they will dominate the low-energy scattering of one of the particles with the bound-state of the remaining two particles. Such states have been studied in several numerical model calculations $[5,8,9]$. There were theoretical searches for Efimov states in atomic and nuclear systems without a clear experimental signature of their occurrence [10-12].

The physical picture underlying such phenomena is related to the unusually large size of these light three-body halo nuclei. The core can be assumed to be structureless $[5,13]$ considering that the radius of the neutron halo is much greater than the radius of the core. The large size scale of the orbit of the outer neutrons in halo nuclei comes from the small neutron separation energies, characterizing a weakly bound few-body system. Thus, the detailed form of the nuclear interaction is not important, which provides the system with universal properties, as long as certain physical scales are known [6]. This situation allows the use of concepts considered in short-range interactions.

In the limit of a zero-range interaction, the three-body system is parameterized by the physical two-body and threebody scales. In the renormalization approach of the quantum mechanical many-body model with the $s$-wave zero-range force, the knowledge of physical information (one from three body and another from two body) can well describe all the low-energy properties of the three-body system [14]. The three-body input can be chosen as the experimental ground state binding energy. All the detailed information about the short-range force, beyond the low-energy two-body observables, is retained in only one three-body physical information, in the limit of the zero-range interaction. The sensitivity of the three-body binding energy to the interaction properties comes from the collapse of the system in the limit of zerorange force. This is known as the Thomas effect [15].

The three-body scale vanishes as a physical parameter if the angular momentum or the symmetries do not allow the simultaneous presence of the particles close to each other. In three-body $p$-wave states, the centrifugal barrier forbid the third particle to be close to the interacting pair (where the pair is supposed to be interacting through the $s$-wave potential). Consequently, the third particle just notices the asymptotic wave of the interacting pair, which is defined by a two-body physical scale. In these states, the system is not sensitive to the three-body scale. The observables of the three-body system, in states that have nonzero angular momentum, are only determined by two-body scales. We look for special possibilities in the $p$ wave like the virtual state. The trineutron system in the $p$ wave presents a peculiar pole in the second energy sheet $[16,17]$, when the neutron-neutron $(n-n)$ is artificially bound. The value of the pole scales with the binding energy of the fictitious $n-n$ system, as this is the only scale of the three-body system [17]. In principle, the existence of one virtual state in three-body halo nuclei systems in $p$ wave is not forbidden. If such a state exists, it will depend exclusively on the two-body scales namely, the binding energy of the neutron to the core and the $n-n$ virtual state energy.

In this work, we search for the virtual state of the threebody halo nuclei in $p$ waves. We consider the zero-range model that is well defined in $p$ waves. The inputs are the energy of the bound state of the neutron to the core and the $n-n$ virtual state energy. We look for weakly bound $n$-core systems; in particular, we examine ${ }^{12} \mathrm{Be}\left({ }^{10} \mathrm{Be}+2 n\right),{ }^{18} \mathrm{C}$ $\left({ }^{16} \mathrm{C}+2 n\right)$, and ${ }^{20} \mathrm{C}\left({ }^{18} \mathrm{C}+2 n\right)$. The zero-range model equa- 
tion is analytically continued to the second energy sheet in the complex plane. There, we search the solution of the homogeneous equation. In the case of Borromean halo nuclei, such as ${ }^{11} \mathrm{Li}$, our method does not work. However, in this case, to get rid of the virtual state, the ${ }^{10} \mathrm{Li}\left({ }^{9} \mathrm{Li}+n\right)$ is artificially bound, in order to allow the analytical continuation to the second energy sheet through the elastic cut.

The nuclei ${ }^{12} \mathrm{Be},{ }^{18} \mathrm{C}$, and ${ }^{20} \mathrm{C}$ have an interesting nonBorromean nature with strong $n-n$ pairing in the ground state. Specifically, ${ }^{12} \mathrm{Be}$ is $\left\{\mathrm{O}^{+}, 23.6 \mathrm{~ms}, E_{n}=3169 \mathrm{KeV}\right\}$, ${ }^{18} \mathrm{C}$ is $\left\{\mathrm{O}^{+}, 95 \mathrm{~ms}, E_{n}=4180 \mathrm{KeV}\right\}$, and ${ }^{20} \mathrm{C}$ is $\left\{\mathrm{O}^{+}, ?, E_{n}=3340 \mathrm{KeV}\right\}$, where the first number is the spin parity of the ground state, the second is the mean lifetime, and the third is the neutron separation energy. The lifetime of ${ }^{20} \mathrm{C}$, shown by a question mark, is not available. The numbers should be compared to the one-neutron-less isotopes, ${ }^{11} \mathrm{Be},{ }^{17} \mathrm{C}$, and ${ }^{19} \mathrm{C}$, respectively, given by $\left\{1 / 2^{+}, 13.81 \mathrm{~ms}\right.$, $\left.E_{n}=504 \mathrm{KeV}\right\}, \quad\left\{?, \quad 193 \mathrm{~ms}, \quad E_{n}=729 \mathrm{KeV}\right\}, \quad$ and $\left\{5 / 2^{+}\left(1 / 2^{+}\right), ?, E_{n}=160(530) \mathrm{KeV}\right\}$. The number in the parentheses, in ${ }^{19} \mathrm{C}$, refers to the recent measurement of Nakamura et al. [18]. Again, the question marks refer to results that are not available. The above nuclei are used to determine the neutron-core binding energies in our calculations. Note that the $n-n$ pairing energies, $\Delta_{n n}$, are in the range 2260 $\leqslant \Delta_{n n} \leqslant 3400 \mathrm{KeV}$. In our calculation of the $p$-wave virtual state, the pairing is taken to be inoperative. The only energy scales left are the neutron-core binding energy $\left(E_{n c}\right)$ and the $n-n$ virtual state energy $\left(E_{n n}\right)$ in the $p$-wave three-body virtual state (pygmy dipole state).

As the input energies are fixed by the renormalized model, the generality of the present conclusions will not be affected by a potential that is more realistic. The corrections due to the Pauli principle, between the halo and the core neutrons, affect essentially the ground state. They are weakened in the $p$-wave state due to the centrifugal barrier. We have to consider that this is a short-range phenomenon that occurs for distances less than the core size (about $\approx 3 \mathrm{fm}$ for light-halo nuclei). We believe that our results are valid even in the case where the spin of the core is nonzero. The results show weak dependence on the mass difference of the particles, in a sense explained together with the numerical results. This is enough to suggest that the dependence on the details of the interactions cannot be larger.

In other contexts, the three-nucleon system has been studied with zero-range force models [11]. Such models succeeded in explaining the qualitative properties of the threenucleon system and described the known correlations between three-nucleon observables. The universality in the three-nucleon system means the independence of the correlations to the details of the short-range nucleon-nucleon potentials [11].

Here, we use a notation appropriate for halo nuclei, $n$ for neutron and $c$ for core. We would like to point out, however, that our approach is applicable to any three-particle system that interacts via $s$-wave short-range interactions, where two of the particles are identical. The $s$-wave interaction for the $n-c$ potential is justified in the present analysis because the $p$-wave virtual state (if it exists) should have a very small energy. It would be sensitive to the properties of the zero angular momentum two-particle state in the relative coordinates. It was also observed in Ref. [19] (when discussing ${ }^{11} \mathrm{Li}$ ) that even the three-body wave function with an $s$-wave $n-n$ correlation produces a ground state of the halo nuclei with two or more shell-model configurations.

The energies of the two particle subsystem, $E_{n n}$ and $E_{n c}$, can be virtual or bound. However, the extension to the second energy sheet will be done through the cutting of the elastic scattering of the neutron and the bound neutron-core subsystem. Thus, we are going to use the value of the virtual state energy, $E_{n n}=143 \mathrm{KeV}$, and the binding energy of the neutron to the core $E_{n c}$ in our calculations. We vary the core mass to study the light halo nuclei like ${ }^{11} \mathrm{Li},{ }^{12} \mathrm{Be},{ }^{18} \mathrm{C}$, and ${ }^{20} \mathrm{C}$.

The zero-range three-body integral equation, for the bound state of two identical particles and a core, is written as a generalization of the three-boson integral equation [20]. It is composed of two coupled integral equations, in close analogy to the case of the $s$-wave separable potential model presented in Ref. [13]. The antisymmetrization of the two outer neutrons is satisfied because the spin couples to zero [5]. In our approach the potential form factors and the corresponding strengths are replaced in the renormalization procedure by the two-body binding energies $E_{n n}$ and $E_{n c}$. In the case of bound systems, these quantities are the separation energies. We distinguish these two cases by the following definition:

$$
K_{n n} \equiv-\sqrt{E_{n n}}, \quad K_{n c} \equiv \sqrt{E_{n c}}
$$

where + refers to bound and - to virtual state energies. Our units will be such that $\hbar=1$ and the nucleon mass, $m_{n}=1$.

After partial wave projection, the $l$-wave coupled integral equations for the three-body system consisting of two neutrons and a core $(n-n-c)$ are

$$
\begin{aligned}
\chi_{n n}^{l}(q)= & 2 \tau_{n n}\left(q ; E ; K_{n n}\right) \int_{0}^{\infty} d k G_{1}^{l}(q, k ; E) \chi_{n c}^{l}(k), \\
\chi_{n c}^{l}(q)= & \tau_{n c}\left(q ; E ; K_{n c}\right) \int_{0}^{\infty} d k\left[G_{1}^{l}(k, q ; E) \chi_{n n}^{l}(k)\right. \\
& \left.+A_{c} G_{2}^{l}(q, k ; E) \chi_{n c}^{l}(k)\right],
\end{aligned}
$$

where

$$
\begin{aligned}
\tau_{n n}\left(q ; E ; K_{n n}\right) & =\frac{1}{\pi}\left[\sqrt{E+\frac{A_{c}+2}{4 A_{c}} q^{2}}-K_{n n}\right]^{-1}, \\
\tau_{n c}\left(q ; E ; K_{n c}\right)= & \frac{1}{\pi}\left(\frac{A_{c}+1}{2 A_{c}}\right)^{3 / 2} \\
& \times\left[\sqrt{E+\frac{A_{c}+2}{2\left(A_{c}+1\right)} q^{2}}-K_{n c}\right]^{-1},
\end{aligned}
$$




$$
\begin{aligned}
G_{1}^{l}(q, k ; E)= & 2 A_{c} k^{2} \\
& \times \int_{-1}^{1} d x \frac{P_{l}(x)}{2 A_{c}\left(E+k^{2}\right)+q^{2}\left(A_{c}+1\right)+2 A_{c} q k x}, \\
G_{2}^{l}(q, k ; E)= & 2 k^{2} \\
& \times \int_{-1}^{1} d x \frac{P_{l}(x)}{2 A_{c} E+\left(q^{2}+k^{2}\right)\left(A_{c}+1\right)+2 q k x} .
\end{aligned}
$$

In the above equations, $A_{c}$ is the core mass number and $E$ is the modulus of the energy of the three-body halo state. As we are concerned with nonzero angular momenta, the Thomas collapse does not appear and the momentum integration can be extended to infinity. For $l>0$ the short-range three-body scale is not effective while the renormalization of the Faddeev equations is necessary for $l=0$. In the renormalization procedure, for $l=0$, a momentum scale is introduced usually as a subtraction point in the integral equations [14]. Such a momentum scale qualitatively represents the inverse of the interaction radius [11]. The subtraction point goes to infinity as the radius of the interaction decreases. So, the three-body model is renormalizable for $l=0$, requiring only one three-body observable to be fixed [14] once the twobody low-energy physical informations are given. The scheme is invariant under renormalization group transformations. However, for $l>0$, the original equations, as given by Eqs. (2) and (3), are well defined and the three-body observables are completely determined by the two-body physical scales related to $K_{n n}$ and $K_{n c}$.

The analytic continuation of the scattering equations to the second energy sheet, for separable potentials, was extensively discussed by Glöckle [16], and, in the zero-range three-body model [20], by Frederico et al. [21]. The analytical continuation is performed through the two-body elastic scattering cut due to the neutron scattering on the bound neutron-core subsystem. In Eq. (5), the elastic scattering cut comes through the pole of the neutron-core elastic scattering amplitude. We then perform the analytic continuation of Eqs. $(2)-(7)$ to the second energy sheet. The spectator function $\chi_{n c}^{l}(k)$ is substituted by $\chi_{n c}^{l}(k) /\left[B_{v}+\left[A_{c}+2 / 2\left(A_{c}+1\right)\right] k^{2}\right]$, where $B_{v} \equiv E_{v}-E_{n c}$ and $E_{v}$ is the modulus of the virtual state energy. The resulting coupled equations, in the second energy sheet, are given by

$$
\begin{aligned}
\chi_{n n}^{l}(q)= & 2 \tau_{n n}\left(q ; E_{v} ; K_{n n}\right) \\
& \times\left[\frac{2 i\left(A_{c}+1\right)}{\pi q\left(A_{c}+2\right)} G_{1}^{l}\left(q,-i k_{v} ; E_{v}\right) \chi_{n c}^{l}\left(-i k_{v}\right)\right. \\
& \left.+\int_{0}^{\infty} d k \frac{G_{1}^{l}\left(q, k ; E_{v}\right) \chi_{n c}^{l}(k)}{B_{v}+\frac{A_{c}+2}{2\left(A_{c}+1\right)} k^{2}}\right],
\end{aligned}
$$

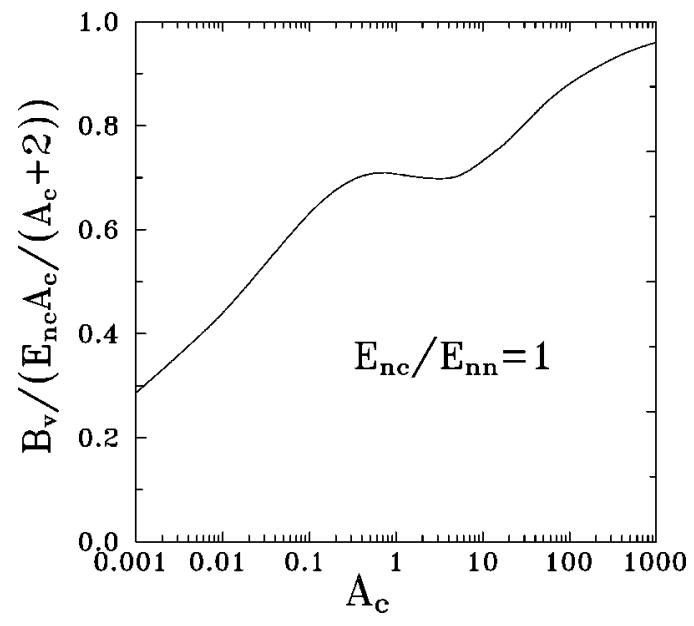

FIG. 1. Scaling plot of $A_{c}+2 / A_{c} B_{v} / E_{n c}$ as a function of the core mass number $A_{c}$ for $E_{n c}=E_{n n} . B_{v} \equiv E_{v}-E_{n c}$, where $E_{v}$ is the $p$-wave virtual state energy, and $E_{n c}$ is the bound state energy of the neutron-core subsystem. $E_{n n}$ is the virtual state energy of two neutrons.

$$
\begin{aligned}
\chi_{n c}^{l}(q)= & \bar{\tau}_{n c}\left(q ; E_{v} ; K_{n c}\right) \frac{2 i A_{c}\left(A_{c}+1\right)}{\pi q\left(A_{c}+2\right)} G_{2}^{l} \\
& \times\left(q,-i k_{v} ; E_{v}\right) \chi_{n c}^{l}\left(-i k_{v}\right) \bar{\tau}_{n c}\left(q ; E_{v} ; K_{n c}\right) \\
& \times \int_{0}^{\infty} d k\left(G_{1}^{l}\left(k, q ; E_{v}\right) \chi_{n n}^{l}(k)\right. \\
& \left.+\frac{A_{c} G_{2}^{l}\left(q, k ; E_{v}\right) \chi_{n c}^{l}(k)}{A_{c}+2} k_{v}^{2}\right)
\end{aligned}
$$

where the on-energy-shell momentum at the virtual state is $k_{v}=\sqrt{2\left(A_{c}+1\right) / A_{c}+2 B_{v}}$, and

$$
\bar{\tau}_{n c}\left(q ; E ; K_{n c}\right)=\frac{1}{\pi}\left(\frac{A_{c}+1}{2 A_{c}}\right)^{3 / 2}\left[\sqrt{E+\frac{A_{c}+2}{2\left(A_{c}+1\right)} q^{2}}+K_{n c}\right] .
$$

The cut of the elastic amplitude, given by the exchange of the core between the different possibilities of the bound coreneutron subsystems, is near the physical region of the pole related to the virtual state due to the small value of $E_{n c}$. Corresponding to the first term of the right-hand side of Eq. (9), the cut is obtained from the imaginary values of $k$ between the extreme poles of the free three-body Green's function, $G_{2}^{l}\left(q, k, E_{v}\right)$, given by Eq. (7):

$$
2 A_{c} E+\left(q^{2}+k^{2}\right)\left(A_{c}+1\right)+2 q k x=0,
$$

where $-1<x<1, q=k=-i k_{c u t}$, and $E=\left[A_{c}+2 / 2\left(A_{c}\right.\right.$ $+1)] k_{c u t}^{2}+E_{n c}$. So, the cut is found at values of $E$ satisfying

$$
2 \frac{A_{c}+1}{A_{c}} E_{n c}>E>2 \frac{A_{c}+1}{A_{c}+2} E_{n c} .
$$




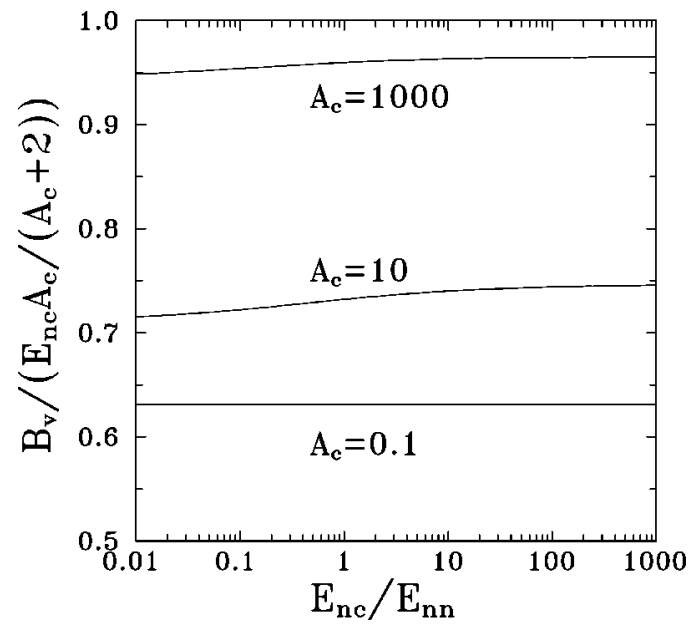

FIG. 2. Scaling plot of $A_{c}+2 / A_{c} B_{v} / E_{n c}$, as a function of $E_{n c} / E_{n n}$, for $A_{c}=0.1,10$, and 100 . The definitions are the same as in Fig. 1.

The virtual state energy $E_{v}$, in the second energy sheet, is found between the scattering threshold and the cut, $E_{v}$ $<2\left(A_{c}+1 / A_{c}+2\right) E_{n c}$, such that $B_{v} \equiv E_{v}-E_{n c}<\left(A_{c} / A_{c}\right.$ $+2) E_{n c}$.

In the limit of zero-ranged interaction for $p$ waves, the only physical scales of the three-body system are $E_{n n}$ and $E_{n c}$. This implies that $B_{v}=E_{n c} \mathcal{F}\left(E_{n c} / E_{n n}, A_{c}\right)$, where $\mathcal{F}$ is a scaling function to be determined by the solution of Eqs. (8) and (9). However, due to the proximity of the cut to the scattering threshold, it is reasonable to believe that it should have major importance in the formation of the virtual state, with $\mathcal{F}\left(E_{n c} / E_{n n}, A_{c}\right)$ being roughly independent of the ratio $E_{n c} / E_{n n}$. Another consequence of the dominance of the cut in the virtual state energy, is that the ratio $B_{v}\left(A_{c}\right.$ $+2) /\left(E_{n c} A_{c}\right)$ should have a soft dependence on $A_{c}$.

In Fig. 1, the results of the virtual state energy are shown in the form of the ratio $B_{v}\left(A_{c}+2\right) /\left(E_{n c} A_{c}\right)$ as a function of the core mass $A_{c}$ for $E_{n c}=E_{n n}$. The same numerical values were chosen for the $n-n$ virtual and $n-c$ bound state energies. The calculations are presented for extreme variations of $A_{c}$, from 0.001 to 1000 , while the ratio was changed by a factor equal to three. The other characteristic of the virtual state is the approximate independence of $B_{v}\left(A_{c}\right.$ $+2) /\left(E_{n c} A_{c}\right)$ on the ratio $E_{n c} / E_{n n}$. This is confirmed in Fig. 2, where the calculations were performed for values of $E_{n c} / E_{n n}$ between 0.01 and 1000 .
TABLE I. $p$-wave virtual state energies $E_{v}$ of light-halo nuclei. The binding energies of the neutron to the core $E_{n c}$ are obtained from the central values given in Ref. [7]. For ${ }^{20} \mathrm{C}$, we also use another value for the binding energy of a neutron to ${ }^{19} \mathrm{C}\left(E_{n c}\right.$ $=530 \pm 130 \mathrm{KeV}$ ) from Ref. [18]. $A_{c}$ is the core mass number and $B_{v} \equiv E_{v}-E_{n c}$.

\begin{tabular}{lrcrc}
\hline \hline Nucleus & $A_{c}$ & $E_{n c}(\mathrm{KeV})$ & $E_{v}(\mathrm{KeV})$ & $B_{v}\left(A_{c}+2\right) /\left(E_{n c} A_{c}\right)$ \\
\hline${ }^{11} \mathrm{Li}$ & 9 & 50 & 79.54 & 0.7221 \\
${ }^{12} \mathrm{Be}$ & 10 & 504 & 813.65 & 0.7373 \\
${ }^{18} \mathrm{C}$ & 16 & 729 & 1227.54 & 0.7694 \\
${ }^{20} \mathrm{C}$ & 18 & 162 & 274.36 & 0.7706 \\
${ }^{20} \mathrm{C}$ & 18 & 530 & 900.30 & 0.7763 \\
\hline \hline
\end{tabular}

The three-body halo nuclei ${ }^{11} \mathrm{Li},{ }^{12} \mathrm{Be},{ }^{18} \mathrm{C}$, and ${ }^{20} \mathrm{C}$ have the $p$-wave virtual state. In the case of ${ }^{11} \mathrm{Li}$, we have artificially changed the virtual state of ${ }^{10} \mathrm{Li}$ to a bound state in order to give the reader one value for a three-body virtual state when the binding energy of the neutron to the core is about a few tenths of $\mathrm{KeV}$. In Table I, we present our results. The $p$-wave virtual state energy scales with the binding energy of the neutron to the core. The values of the virtual state energies are close to 1.7 times the values of $E_{n c}$.

In summary, we have discussed universal aspects of $p$-wave virtual states of three-body halo nuclei in the limit of a zero-range interaction. As shown, the values of the $p$-wave virtual state energies are determined by the existence of scaling properties of the three-body $p$-wave virtual state energies, with respect to the $n-n$ virtual and $n-c$ bound state energies. Our conclusion is that the scaling function, $\mathcal{F}\left(E_{n c} / E_{n n}, A_{c}\right)$, which gives the virtual state energy as $E_{v}$ $=E_{n c}\left[1+\mathcal{F}\left(E_{n c} / E_{n n}, A_{c}\right)\right]$, roughly does not depend on the ratio $E_{n c} / E_{n n}$; it is almost entirely determined by $A_{c}$. From the knowledge of $E_{n c}$, we have obtained the $p$-wave virtual state energies for ${ }^{12} \mathrm{Be},{ }^{18} \mathrm{C}$, and ${ }^{20} \mathrm{C}$, which came out to be about 1.7 times the corresponding neutron-core binding energies. These threshold dominated excited states, commonly called "pygmy resonances," are, therefore, not resonances at all. They correspond to a manifestation of predominantly dipole final state interactions just as in the two-body case of the most well-known halo nucleus, the deuteron [22].

Our thanks to Fundação de Amparo à Pesquisa do Estado de São Paulo (FAPESP) and Conselho Nacional de Desenvolvimento Científico e Tecnológico $(\mathrm{CNPq})$ of Brazil for partial support.
[1] V. Efimov, Phys. Lett. 33B, 563 (1970).

[2] I. Tanihata, J. Phys. G 22, 157 (1996).

[3] C.A. Bertulani, L.F. Canto, and M.S. Hussein, Phys. Rep. 226, 281 (1993).

[4] P.G. Hansen, A.S. Jensen, and B. Jonson, Annu. Rev. Nucl. Part. Sci. 45, 591 (1995); M.V. Zhukov, B.V. Danilin, D.V. Fedorov, J.M. Bang, I.S. Thompson, and J.S. Vaagen, Phys. Rep. 231, 151 (1993).

[5] D.V. Fedorov and A.S. Jensen, Phys. Rev. Lett. 25, 4103
(1993); D.V. Fedorov, A.S. Jensen, and K. Riisager, Phys. Rev. Lett. 73, 2817 (1994); D.V. Fedorov, E. Garrido, and A.S. Jensen, Phys. Rev. C 51, 3052 (1995).

[6] A.E.A. Amorim, L. Tomio, and T. Frederico, Phys. Rev. C 56, R2378 (1997).

[7] G. Audi and A.H. Wapstra, Nucl. Phys. A595, 409 (1995); ftp://csnftp.in2p3.fr/AMDC/masstables/

[8] A.T. Stelbovics and L.R. Dodd, Phys. Lett. 39B, 450 (1972); A.C. Antunes, V.L. Baltar, and E.M. Ferreira, Nucl. Phys. 
A265, 365 (1976).

[9] S.K. Adhikari and L. Tomio, Phys. Rev. C 26, 83 (1982); S.K. Adhikari, A.C. Fonseca, and L. Tomio, ibid. 26, 77 (1982).

[10] T.K. Lim, K. Duffy, and W.C. Damest, Phys. Rev. Lett. 38, 341 (1977); H.S. Huber, T.K. Lim, and D.H. Feng, Phys. Rev. C 18, 1534 (1978).

[11] V. Efimov, Comments Nucl. Part. Phys. 19, 271 (1990); and references therein.

[12] T. Frederico, A. Delfino, A.E.A. Amorim, and L. Tomio, Phys. Rev. A 60, R9 (1999).

[13] S. Dasgupta, I. Mazumdar, and V.S. Bhasin, Phys. Rev. C 50, 550 (1994).

[14] S. K. Adhikari, T. Frederico, and I.D. Goldman, Phys. Rev.
Lett. 74, 487 (1995); S.K. Adhikari and T. Frederico, Phys. Rev. Lett. 74, 4572 (1995).

[15] L.H. Thomas, Phys. Rev. 47, 903 (1935).

[16] W. Glöckle, Phys. Rev. C 18, 564 (1978).

[17] A. Delfino and T. Frederico, Phys. Rev. C 53, 62 (1996).

[18] T. Nakamura et al., Phys. Rev. Lett. 83, 1112 (1999).

[19] M. Zinser et al., Phys. Rev. Lett. 75, 1719 (1995).

[20] G.V. Skornyakov and K.A. Ter-Martirosian, Zh. Éksp. Teor. Fiz. 31, 775 (1957) [Sov. Phys. JETP 4, 648 (1957)].

[21] T. Frederico, I.D. Goldman, and A. Delfino, Phys. Rev. C 37, 497 (1988).

[22] M.S. Hussein, C.-Y. Lin, and A.F.R. de Toledo Piza, Z. Phys. A 355, 165 (1996). 